\title{
Матричные КМОП-фотоприемники: современное состояние и перспективы развития
}

\author{
А. Сафонов, к. т. н. ${ }^{1}$
}

УДК 621.383 | ВАК 05.27 .01

\begin{abstract}
Наряду с процессорами и схемами памяти КМОП-фотоприемники - наиболее динамично развивающийся сектор полупроводниковой индустрии. Они определяют параметры систем визуализации как в индустриальных приложениях, так и в потребительской электронике. Основными областями применения устройств являются автомобильная промышленность, системы искусственного интеллекта и видеонаблюдения, смартфоны и даже игрушки. Сопутствующий прогресс в технологии изготовления 3D фотоприемных устройств значительно повлиял на развитие технологии сборки всех типов электронных модулей.
\end{abstract}

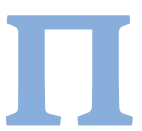
о уровню использования новейших технологий и темпам роста продаж КМОП-фотоприемники выходят на первые позиции в полупроводниковой индустрии, опережая традиционно лидирующие сегменты - память и процессоры - благодаря высокому спросу на чипы для получения изображений во множестве приложений: мобильных телефонов, смартфонов, планшетов, автомобилей, медицинских приборов, приборов технического зрения. Лидерами в данном сегменте стали фотоприемные модули, изготовленные по 3D-технологии. То, что прежде было для потребителя микросхемой, то есть полупроводниковым изделием, помещенным в корпус, снабженным выводами и предназначенным для выполнения специальной функции, теперь представляет собой интегрированные структуры, содержащие функционально отличные элементы: фотоприемники с элементами оптики, память, логические и аналоговые схемы, высокоразрядные быстродействующие АЦП и процессоры. Все они изготавливаются в едином технологическом цикле на базе КМОПтехнологии. Объем продаж фотоприемных микросхем в 2017 году составил более 13,9 млрд долл., рост рынка превышает 10\%. По прогнозам, к 2023 году объем продаж превысит 23 млрд долл. Годовой прирост в 9,3\% будет обеспечен в основном за счет реализации дополнительных камер, интегрированных в смартфоны для поддержания функций оптического зума (трансфокации) биометрии и 3D-интеграции.

Лидерами рынка являются 13 фирм во главе с Sony, которой принадлежит доля в 42\% (рис. 1). Основной объем

ФГУП «ниИ физических проблем им. Ф. В. Лукина», начальник отдела. asaf.niifp@gmail.com. выпуска составляют схемы для потребительских, промышленных и медицинских приложений, доля производителей изделий для обороны и специальных задач, в том числе научных, не превышает 5\%, хотя эти изделия в ряде случаев отличаются рекордными техническими характеристиками.

Такие достижения стали возможными благодаря совершенствованию составляющих процесса изготовления КМОП-фотоприемников: конструкции элементов, архитектуры, технологии.

Потребительские свойства КМОП-фотоприемников определяются набором параметров, которые можно разделить на основные и вторичные. Выбор вторичных параметров подразумевает компромисс, поскольку максимальное их значение нельзя получить одновременно. Три основных параметра: качество изображения, быстродействие (максимальное количество кадров в секунду) и разрешение. Второй набор параметров (оценивает потребитель): функциональность камеры или датчика, где конкурирующие функции требуют сложных решений. Примерами этих параметров являются такие функции, как оконная обработка и энергопотребление. Компромиссные варианты касаются стоимости, доходности, надежности и других особенностей, связанных с изготовлением устройства формирования изображений [1].

\section{ПАРАМЕТРЫ ФОТОЧУВСТВИТЕЛЬНОЙ ЯЧЕЙКИ}

Основной элемент фотоприемника как датчика - фоточувствительная ячейка (пиксель), в которой излучение преобразуется в электрический сигнал. Пиксель в современном КМОП-фотоприемнике состоит из фоточувствительной области и управляющих активных элементов затворов и транзисторов. 


\section{Коэффициент заполнения}

Существует обратная зависимость между количеством транзисторов в пикселе и коэффициентом его заполнения. С одной стороны, коэффициент заполнения (процент чувствительной к свету области в пикселе) непосредственно влияет на чувствительность датчика и на соотношение сигнал/шум (S/N) захваченного изображения. С другой стороны, наличие большего количества транзисторов в пикселе обеспечивает доступ к дополнительным функциям, таким как глобальный затвор (кадровое считывание) и коррелированная двойная выборка (CDS), повышающим качество изображения. Примеры двух основных типов пикселей приведены на рис. 2.

В ячейке первого типа (рис. 2а) область приема излучения совмещена с областью хранения. Такие ячейки используются в режиме построчного считывания (Rolling Shutter). Более высокое разрешение можно получить в ячейке с фотозатвором (рис. 26), разделяющим области приема излучения и хранения заряда. Такие ячейки используются в режиме кадрового считывания.

\section{Угол приема света}

Чтобы минимизировать влияние увеличенного количества транзисторов на пиксель, в большей части КМОПдатчиков изображения используются микролинзы. Благодаря этому компенсируется часть потерянной из-за увеличения числа транзисторов энергии в пикселе, однако при этом уменьшается угол приема света в пикселе. Применение микролинз - это компромисс между количеством транзисторов в пикселе и качеством изображения.

\section{Зарядовая емкость и максимальное время экспозиции}

Зарядовая емкость - количество заряда, который может накапливаться в фоточувствительной области во время экспозиции. Этот важнейший параметр определяет динамический диапазон, зависит от геометрических размеров ячейки, напряжения питания и коэффициента заполнения.

\section{Минимальное время экспозиции, формат и энергопотребление}

Минимальное время экспозиции напрямую определяет максимальную практическую скорость работы устройства формирования изображения. Датчик, который не имеет оптимальной конфигурации, может искажать изображения при малом времени экспозиции и не искажать при более длительных экспозициях. В конструкции КМОП-датчика минимальное время экспозиции определяется скоростью распространения сигнала внутри датчика - от пикселя к выходу. Время увеличивается по мере роста числа элементов, при этом повышается и энергопотребление.

\section{Минимальный уровень шума}

Минимально достижимый уровень шума в пикселе очень важен при низких уровнях освещенности. Сложная схема пикселя и увеличенное количество ступеней обработки сигнала может отрицательно сказаться на уровне шума датчика. Существующие методы уменьшения уровня шума, например, коррелированная двойная выборка, требуют дополнительной памяти в архитектуре пикселей, что сокращает объем для сбора света и хранения сигналов и ограничивает оптическую эффективность. 


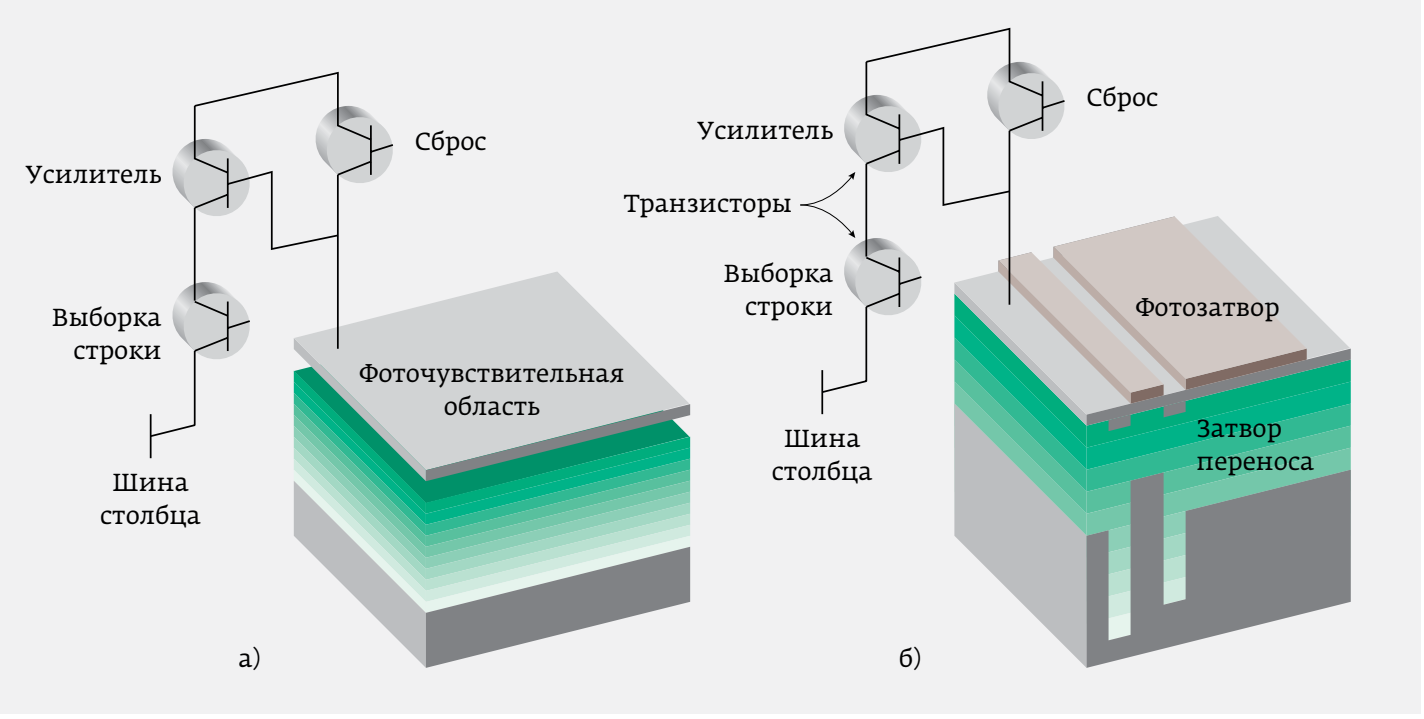

Рис. 2.

Основные типы пикселей: с фотозатвором (а), с фотозатвором и затвором переноса (б)

\section{СОВРЕМЕННАЯ АРХИТЕКТУРА КМОП-ФОТОПРИЕМНИКА \\ Качество изображения}

Качество изображения определяется преимущественно техническими характеристиками фотоприемной ячейки пикселя. В КМОП-фотоприемнике пиксель представляет собой конструкцию из оптического канала, по которому излучение попадает на преобразователь "излучение-заряд", элементов выборки и первичной обработки сигнала.

Конструкция современного пикселя приведена на рис. 3. Оптический канал образован микролинзой, цветовым фильтром и антиотражающим покрытием. Преобразователем излучения служит фотодиод структуры $\mathrm{p}^{+}-n-\mathrm{p}$. В состав пикселя входят четыре транзистора: переноса, сброса, выборки строки и усилительный. Вертикальные границы оптического канала образуются слоями непрозрачного материала (металла или органического слоя), границы в полупроводнике - одним из традиционных способов изоляции, применяемых в КМОП-технологии.

Зарядовая емкость пикселя снижается, а уровень шума увеличивается за счет паразитных периферийныхтоков, собираемых из полупроводниковой области, окружающей пиксель. Для минимизации паразитных токов и шумов в конструкцию пикселя добавляется слой p $^{+}$[2] .

Геометрические размеры пикселя в современных приемниках достигают 1,1 мкм в серийно выпускаемых изделиях и 0,9 мкм - в новых разработках. Метод
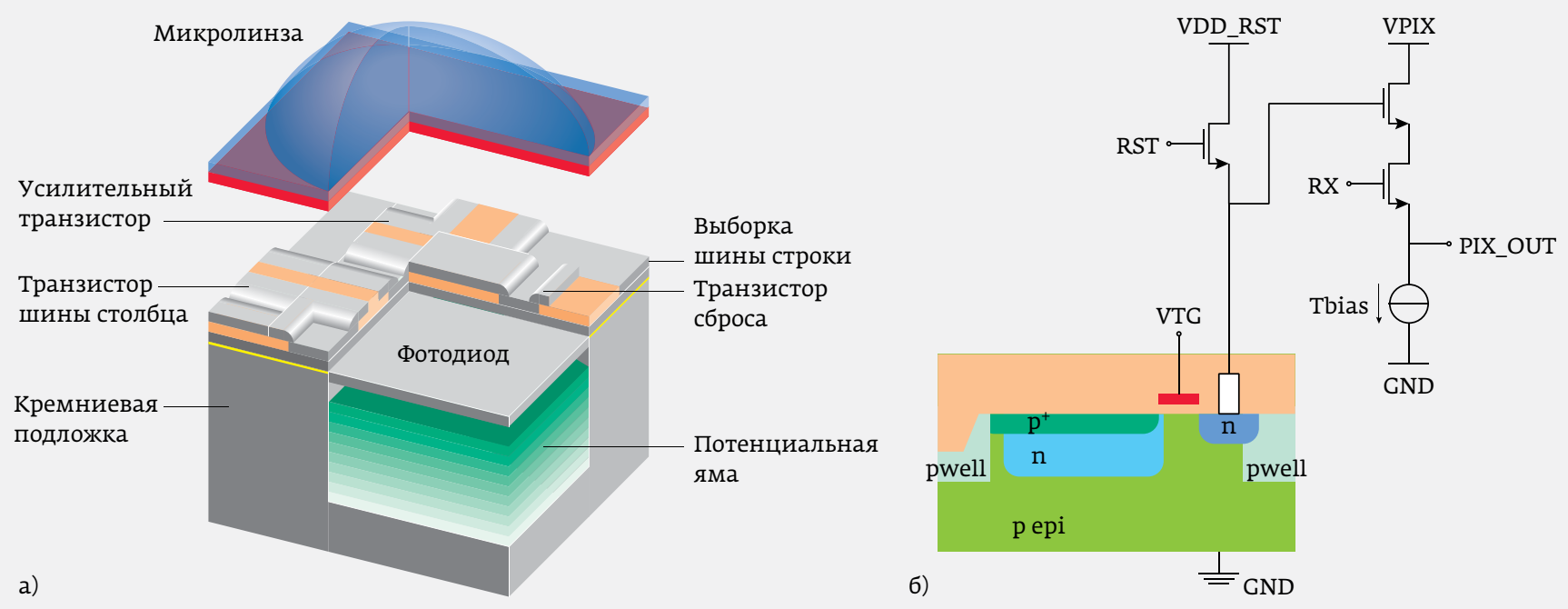

Рис. 3. Современная конструкция (а) и схема (б) активного пикселя 
увеличения коэффициента заполнения состоит в том, чтобы некоторые электронные компоненты (например, транзисторы выборки, сброса и усилительный) стали общими для соседних ячеек. Количество фотодиодов с общими элементами достигает шести благодаря оптимальной топологической конструкции. Опрос фотодиодов производится последовательно при помощи транзистора переноса.

\section{Динамический диапазон и соотношение сигнал / шум}

Два параметра: динамический диапазон и соотношение сигнал/шум связаны между собой. Динамический диапазон - отношение максимального количества накопленного заряда к уровню темнового заряда. Динамический диапазон определяет соотношение между максимальной и минимальной интенсивностью света, которые могут быть обнаружены одновременно.

Это важный параметр для многих приложений в автомобильной, охранной и следящей, медицинской и научной областях. Однако для обычных датчиков изображения характерен ограниченный динамический диапазон: соотношение между самой высокой и самой низкой интенсивностью света, которую можно передавать, составляет, как правило, 1000 :1 или 10000 :1. Этот относительно узкий диапазон приводит к неточному представлению изображений, содержащихобласти с различной интенсивностью света. Для расширения динамического диапазона используется метод многократного захвата с использованием разного времени экспозиции. Изображение при этом формируется обычным путем, но требуется постобработка, приводящая к низкой частоте кадров и другим артефактам (ореолам и размытому изображению).

Другие подходы к увеличению динамического диапазона представляют собой в основном конкретные топологии пикселей для решения конкретных задач. К ним относятся логарифмические пиксели, многомодовые операции, регулировка емкости, частотная и временная работа пикселей и селективное время интеграции [2]. Верхний предел динамического диапазона определяется зарядовой емкостью пикселя, а нижний ограничивается несколькими источниками шума (тепловым, шумом считывания и др.). Тепловой шум обусловлен тепловой генерацией-рекомбинацией электронов и дырок в кремниевой подложке. Эти носители затем собираются фотодетектором так же, как носители, генерируемые путем поглощения фотонов. В астрономии и других областях обычная практика - охлаждение датчика и связанной с ним электроники до криогенных уровней, однако это непрактично для других вариантов применения.

Паразитные токи утечки из-за дефектов вблизи областей изоляции также порождают шум. Для улучшения этих параметров используется конструкция фотодиода типа $\mathrm{p}^{+}-n-p$ (рис. 36). При полном обеднении такой структуры образуется потенциальная яма, в которой накапливается заряд. Стенки потенциальной ямы изолируют полезный заряд от поверхности и объема полупроводника. Таким образом исключаются захват заряда на поверхностных ловушках и связанный с этим шум, а также поверхностная генерация темнового заряда и сбор объемного темнового тока. При подаче напряжения переноса весь накопленный заряд перетекает в узел считывания.

Достигнутые параметры активного пикселя:

- зарядовая емкость - 14000 электронов;

- коэффициент преобразования - 75 мкВ/электрон;

- динамический диапазон-70 дБ;

- темновой ток-1,5пА/см².

В разрезе пиксель представляет собой многослойную конструкцию, состоящую из слоев: линзы, оптические фильтры, активные элементы, разводка, фоточувствительная область (рис. 4). Изолируются пиксели друг от друга различными приемами, освещаются - фронтально или со стороны подложки.

\section{Оптимизация структур пикселей}

Оптимизация структур пикселей, направленная на улучшение его фотоприемных характеристик, предусматривает уменьшение латерального размера пикселя и методы подавления перекрестных помех. На рис. 5а представлена

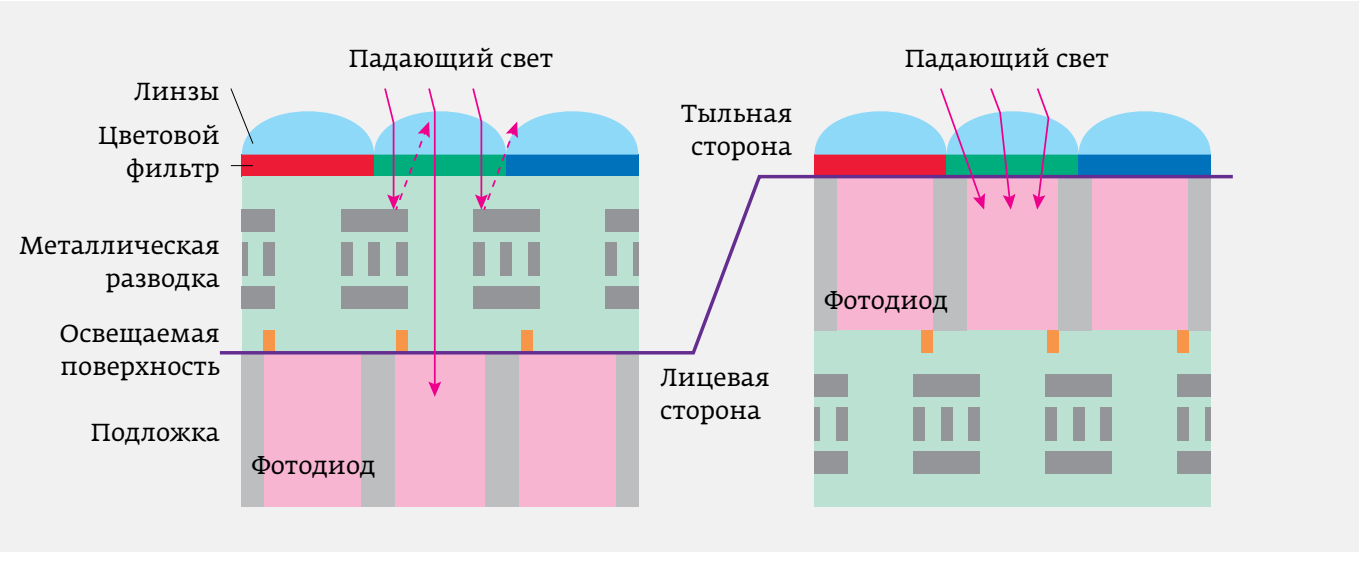

Рис. 4. Разрез активного пикселя при освещении с фронтальной (а) и тыльной (б) стороны 


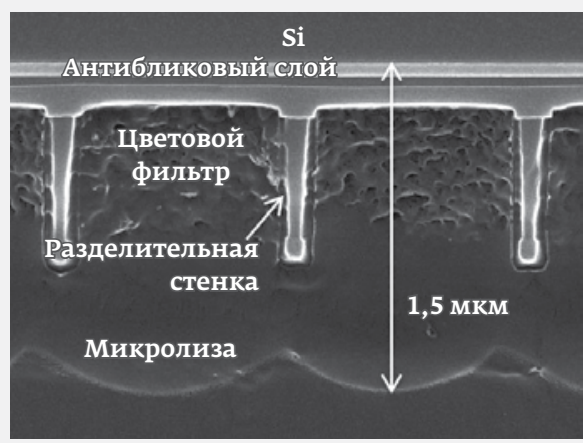

a)

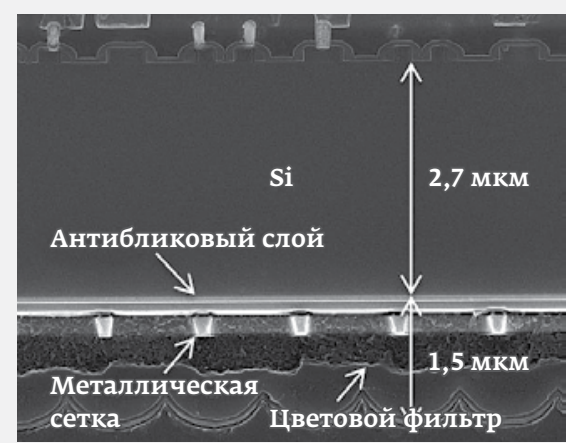

б)

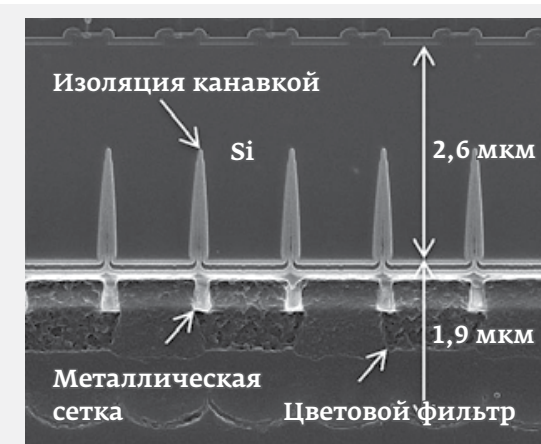

B)

Рис. 5. 3D-конструкции пикселей ведущих мировых производителей: ON Semiconductor AR0842 Clarity+ 1,1 мкM пиксель; Sony IMX214, 1,12 мкм пиксель, встроенные цветовые фильтры (б); Samsung S5K2P2XX, 1,12 мкм пиксель, изоляция канавкой (в)

конструкция [3] с разделительными перегородками между цветовыми фильтрами, фронтально освещенными пикселями. Конструкция адаптирована и для задней подсветки. Структура создает попиксельный оптический волновод размером 1,5 мкм.

Sony и Samsung используют для создания границ между фильтрами металлическую сетку в ячейках с задней подсветкой. В качестве оптической изоляции металлическая сетка более эффективна, чем органические материалы, применявшиеся ранее (рис. 56, в).

Встроенные цветные фильтры Samsung более толстые, поэтому толщина оптического стека составляет 1,9 мкм [4]. Технология встроенных фильтров совмещается с технологией изоляции глубокими канавками (DTI) для уменьшения электрических и оптических перекрестных помех в подложке. Изоляционные канавки первого поколения глубиной около 1,6 мкм в тыльной части подложки толщиной 2,6 мкм. Изоляция канавкой на основе оксида гафния обеспечивает пассивацию поверхности Si и служит в качестве антибликового слоя. Samsung анонсировала дальнейшую работу по полной изоляции каждого пикселя на основе технологии ISOCELL [5].

\section{Конструкция цветовых шаблонов}

Для улучшения цветопередачи используются панхроматические фильтры в сочетании с металлической сеткой.

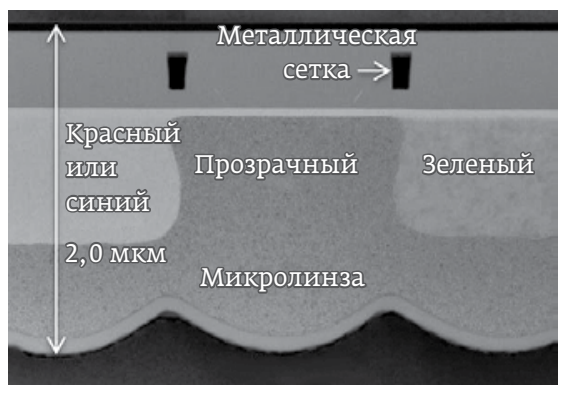

Рис. 6.

Структура фильтра для улучшенной передачи цвета и подавления перекрестных помех
В отличие от фильтров Байера применяется мозаика, состоящая из 25\% красных, 25\% голубых и 50\% прозрачных пикселей (рис. 6).

Представление интенсивности света в изображении не единственный показатель измерения. Нужно различать и другие характеристики света, например, длину волны. Свет с различными длинами волн в видимом спектре классифицируется по цветам, что важно учитывать не только для вариантов потребительского применения, но и для различных технических процессов, контроля качества, а также для случаев обнаружения цветовых оттенков, таких как спектроскопия. В КМОП-датчиках изображения для обнаружения цвета обычно применяются фильтры с различной центральной частотой передачи на разные пиксели, то есть матрицы цветных фильтров (CFA). Поэтому связанные с одним и тем же фильтром пиксели образуют особый цветовой канал в общем массиве. Эти фильтры чаще

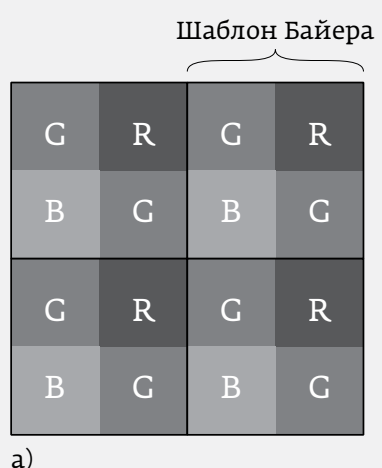

\begin{tabular}{|cc|cc|}
\hline $\mathrm{W}$ & $\mathrm{R}$ & $\mathrm{W}$ & $\mathrm{R}$ \\
$\mathrm{B}$ & $\mathrm{G}$ & $\mathrm{B}$ & $\mathrm{G}$ \\
\hline $\mathrm{W}$ & $\mathrm{R}$ & $\mathrm{W}$ & $\mathrm{R}$ \\
$\mathrm{B}$ & $\mathrm{G}$ & $\mathrm{B}$ & $\mathrm{G}$ \\
\hline
\end{tabular}

б)

Рис. 7. Разные цветовые фильтры: а - широко используемый шаблон Байера, то есть красный-зеленый-зеленый-синий (RGCB); б - конфигурация альтернативного цветового рисунка (RGWB) для увеличения квантовой эффективности датчика 
всего представляют собой органические красители на пути света. Коэффициент их пропускания колеблется от 70 до 90\%, что обеспечивает общий коэффициент пропускания 30-50\% из-за внеполосной передачи по видимому спектру. Поэтому их применение снижает общее поглощение света, то есть квантовую эффективность фотоприемника, и отрицательно сказывается на использовании в условиях низкой освещенности.

В идеальном случае датчик изображения должен различать необходимое количество разных цветов. Однако, согласно теории цвета и механизму обнаружения цвета человеком, требуется только три цветовых фильтра, соответствующих основным цветам, которые представляются взвешенными сложениями или вычитаниями в вычислениях после обработки. Одно из преимуществ использования всего трех цветовыхканалов состоит в том, что уменьшение

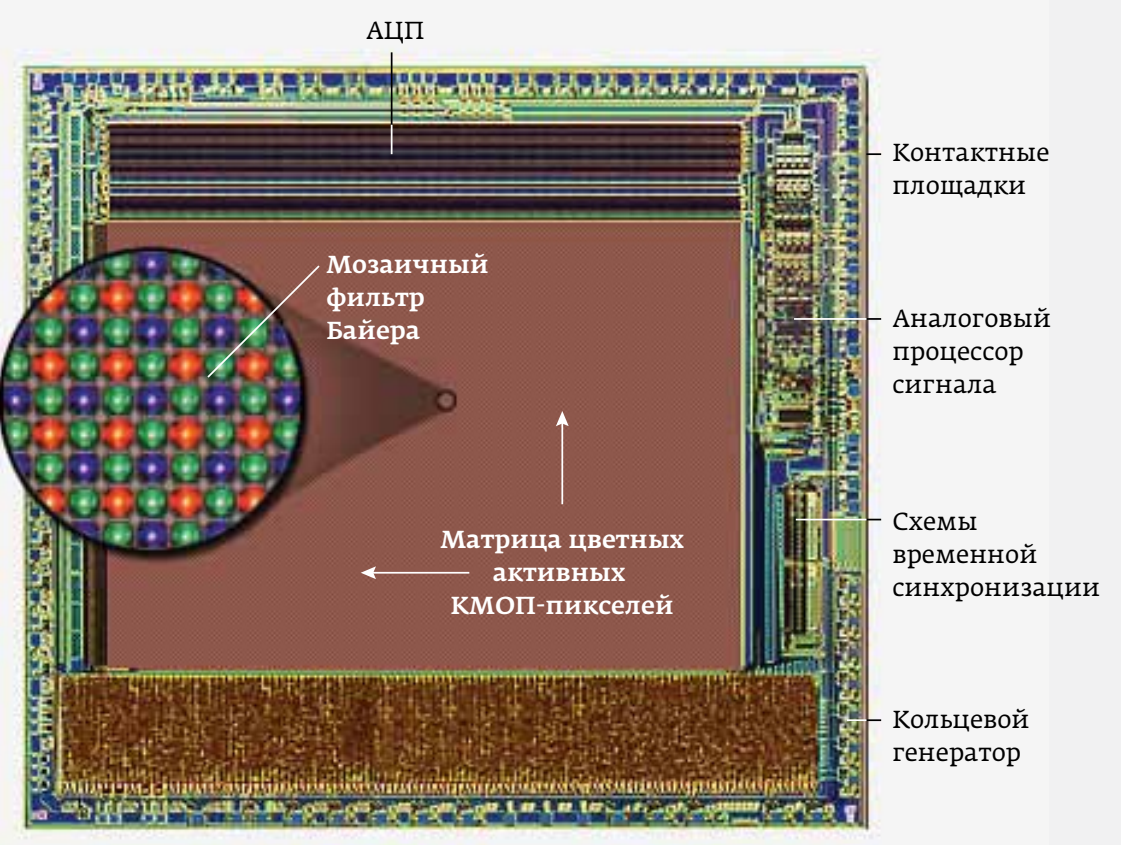

Цифровая логика (интерфейс, тактирование, обработка, выход)

Рис. 8. Латеральное расположение элементов фотоприемника ф. Olympus общего разрешения датчика незначительное. В популярном цветовом шаблоне Байера (RGGB) каждый цветной "пиксель" состоит из массива фотодетекторов 2×2, где два обозначены зеленым каналом, а другие два - синим и красным каналами, как показано на рис. 7а.

Пространственное разрешение датчика изображения из-за этого цветового шаблона составляет 25\% разрешения черно-белого устройства. Некоторые изменения в шаблоне Байера используются для увеличения квантовой эффективности датчика. В шаблонах RGBW / RGBC (рис. 7б) один из зеленых фильтров удаляется для лучшего измерения освещенности, в то время как в шаблоне RCCB зеленые фильтры удаляются полностью, причем зеленая компонента получается обработкой сигнала (вычитанием цветов).

\section{АРХИТЕКТУРА ФОТОПРИЕМНИКА}

До 2010 года все элементы фотоприемников - фоточувствительные пиксели, схемы тактирования и обработки сигнала - располагались латерально на одном кристалле (рис. 8). Такая архитектура не лишена недостатков. Поскольку требования к параметрам возрастали, схемы

\section{Модуль с параллельной перестраиваемой обработкой изображения}

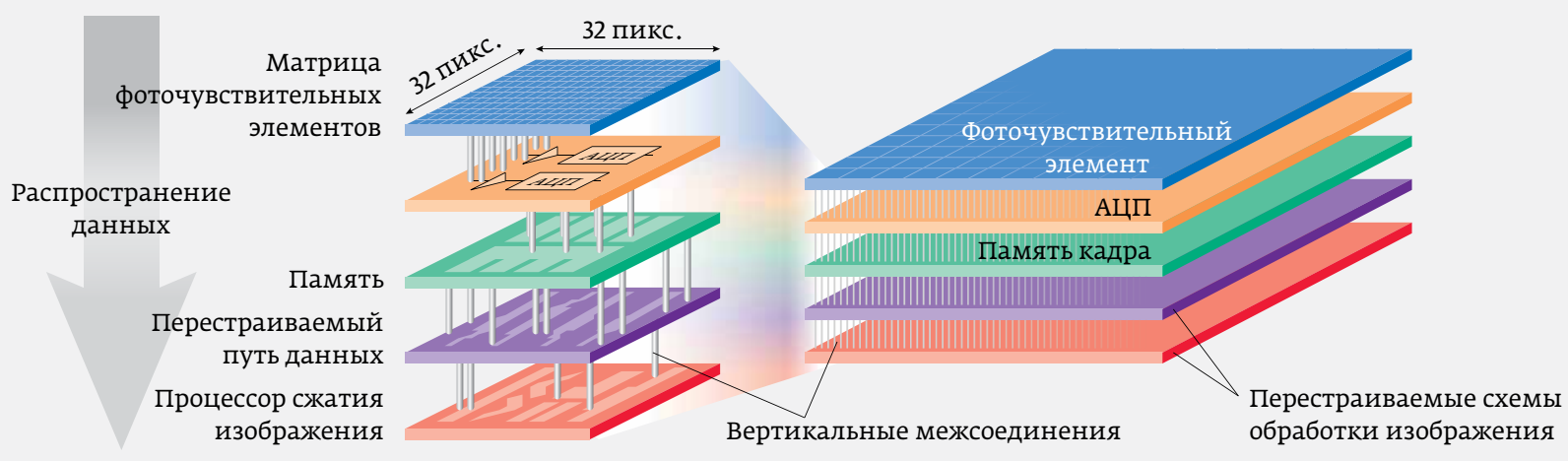

Рис. 9. Функциональное послойное разделение элементов КМОП-фотоприемника 


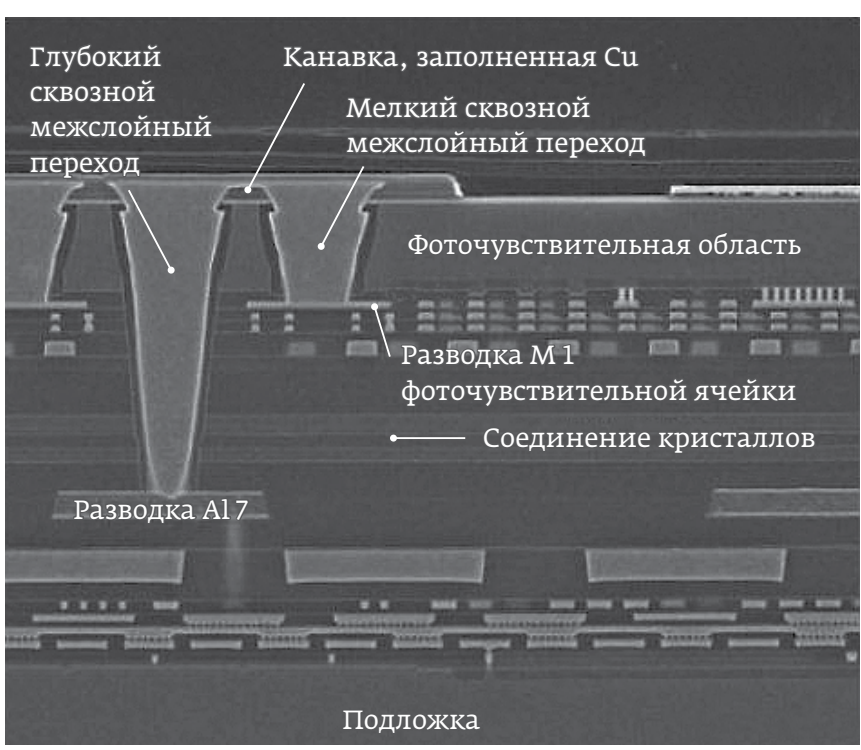

Рис. 10. Разрез 3D-сборки

обработки сигнала усложнялись, занимали на кристалле относительно большую площадь, увеличивалось энергопотребление. Последнее приводило к локальному перегреву отдельных областей кристалла, так как разные функциональные блоки схем обработки потребляют различную мощность. От последовательной передачи сигналов между отдельными блоками невозможно было избавиться, поэтому такие «узкие» места ограничивали быстродействие.

Появление технологии 3D-сборки, пришедшей из области схем микромеханики и памяти (прежде всего флешпамяти), позволило сделать качественный шаг вперед по пути совершенствования функциональныхи технических параметров фотоприемников. Использование 3D-технологии обеспечивает оптимальное разделение функциональных элементов фотоприемника, они размещаются в разных плоскостях и реализуется параллельная обработка сигнала. Близкой к оптимальной, с этой точки зрения, выглядит четырехслойная структура, изображенная на рис. 9. Первый - слой активных пикселей, сигнал с которых попадает на АЦП. После оцифровки (второй слой) данные могут запоминаться (третий слой), что особенно актуально при многократном считывании в течение одного кадра, как в системах измерения разности фаз и квантовых фотоприемниках. Сигнал из памяти в дальнейшем передается для обработки процессором (четвертыйслой).
В настоящее время ведущие производители Sony, Samsung и OmniVision используют 3D-сборку в своих изделиях. На рис. 10 представлена фотография разреза структуры 13-мегапиксельной микросхемы IMX214 фирмы Sony. Фронтально освещаемая матрица пикселей с набором оптических фильтров расположена сверху. Второй слой - схемы обработки.

Геометрические параметры элементов составляют: размер пикселя 1,1×1,1 мкм, толщина одного функционального слоя 60-100 мкм. Дальнейшее совершенствование технологий позволило Sony создать высокоскоростной чип для технического зрения (рис. 11), который имеет 1,27 Мпикселей и быстродействие 1000 кадров в секунду [6]

\section{ОБРАБОТКА ИЗОБРАЖЕНИЙ \\ Скорость получения данных}

В большинстве приложений обработки изображений обычные сцены меняются со временем. Для этого нужно, чтобы датчик фиксировал последовательность изображений (видео) с заданным, в основном фиксированным, периодом времени между этими изображениями (кадрами), определяя оптимальную скорость кадров. Изначально КМОП-фотоприемники использовали строчное считывание, как более простое. Поскольку при этом опрос пикселей в одной строке начинается и заканчивается со смещением по времени относительно начала и окончания опроса пикселей в предыдущей строке, это может генерировать ряд различных эффектов искажения изображения, особенно для быстро изменяющихся сцен, один из которых показан на рис. 12.

Интегрирование изображения в режиме кадрового считывания применяется ко всем пикселям одновременно. Хотя этот метод требует более сложного считывания и пиксельных конструкций, он востребован в таких приложениях, как 3D-съемка, машинное зрение и автомобильная промышленность. В традиционных сканирующих

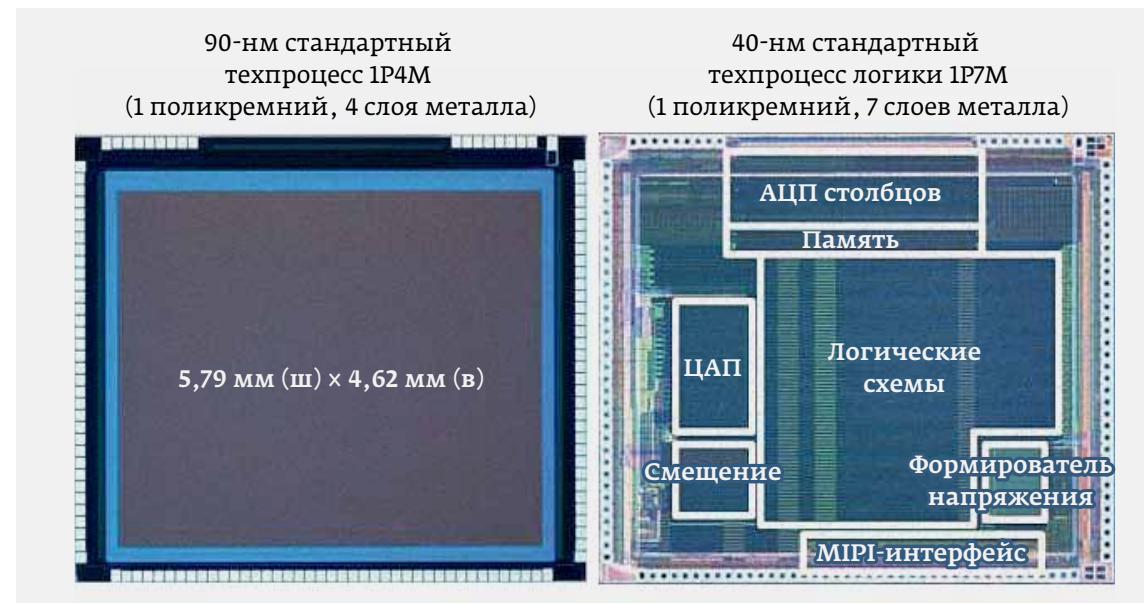

Рис. 11. Микрофотография чипа: а - левая микросхема состоит только из пикселей; б - правый чип содержит большое количество логики 
устройствах считывания используется встроенная память в пикселях для временного хранения информации, преобразованной в свет. Эффективность памяти зависит от эффективности затвора устройства формирования изображения. Цифровые запоминающие устройства, используемые в цифровыхпикселях, более надежны, чем аналоговые, но требуют большой площади в пикселях.

Аналоговая память подвержена утечкам заряда, включая свето-индуцированную утечку тока (паразитную светочувствительность) от p-n-переходов самой памяти (в случае накопительного диода) и от Мдп-переключателей. Некоторые методы уменьшения этой утечки включают экранирование света в области памяти; специальный профиль легирования для поглощения паразитных световых носителей с помощью фотодиода.

Скорость кадров важна для видеоприложений, таких как видео высокой четкости, машинное зрение, трехмерное видение и научные приложения. Если не требуется высокая скорость кадра, обычно используется архитектура с несколькими входами и одним выходом (MISO). Поскольку скорость кадра тесно связана со скоростью обработки на каждом этапе цепочки сигналов (от пиксела до выхода), оптимизируется каждый этап передачи сигнала. Эффективные усовершенствования основаны на перемещении аналого-цифрового преобразования (АЦП) в цепочке сигналов: от исходных аналоговых выходных систем, где цифровизация проводилась вне кристалла, до снабжения АЦП каждого столбца и далее к встроенному в пиксель АЦП. Миграция АЦП как можно ближе к детектору увеличивает цифровой параллелизм и используется для повышения общей пропускной способности.

\section{Достижения в вычислительной визуализации}

Помимо повышения качества изображения расширялись функциональные возможности датчиков, в частности выполнение вычислений в процессе визуализации изображения.

Вычислительная визуализация подразумевает возможность извлечения релевантной информации из сформированных изображений в процессе последующей обработки либо во время их захвата. Варианты вычислительных приложений для визуализации - фокальные манипуляции, например, для фиксации жестов и отслеживания глазного взгляда.

Возможность обработки изображения непосредственно в фотоприемнике обусловлена тем, что технологический процесс его изготовления и принципы конструирования основываются на использовании КМОП-технологии, для которой разработано множество вычислительных конструктивных решений. Однако если обработка выполняется вне массива пикселей, то необработанные данные должны быть собраны, а затем обработаны вне фоточувствительной области, а не на самом пикселе.

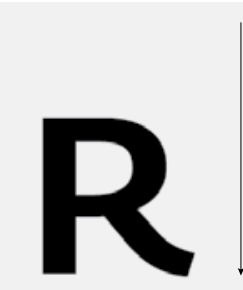

a)

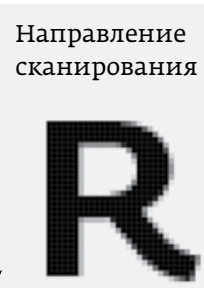

б)

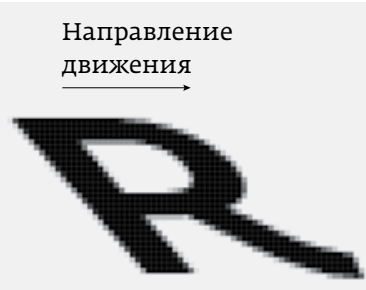

B)
Рис. 12. Примеры влияния режимов экспозиции на быстро меняющиеся изображения. При покадровом считывании изображение (б) сохраняет первоначальную форму (а). Режим с построчным считыванием более простой конструкции сильнее искажает динамическое изображение (в)

Было предложено несколько способов обработки изображений в пикселе. Однако эти схемы с большим количеством транзисторов на пиксель уменьшают оптически чувствительную область и коэффициент заполнения пикселя. Тем не менее даже минимальные изменения конструкции пикселя дают возможность использовать его в качестве компаратора. Если в конструкции на рис. 36 к истоку выходного транзистора вместо заземляющего потенциала приложить опорное напряжение, то по мере изменения его на всем массиве пикселей или их части можно масштабировать участки динамического диапазона, выделять отдельные участки изображения путем их затемнения или увеличения яркости

\section{Фазовое детектирование}

Другой способ обработки сигнала в массиве пикселей. Каждый эффективный пиксель на поверхности КМОП-датчика изображения состоит из двух независимых фотодиодов, которые считывают, каждый самостоятельно, световые сигналы для автофокусировки с фазовым детектированием и совместно - для создания изображения. Для фазового детектирования на всей плоскости изображения левые и правые фотодиоды считывают световые сигналы отдельно. Получаемые при этом смещенные изображения используются для расчета разности фаз. Для повышения точности используется пиксель с четырьмя фотодиодами, два из них, закрытые от света металлическим экраном, выполняют роль опорных. Площадь пикселя равна 4,1 мкм² [7].

\section{Сжатие видеоинформации}

Во многих приложениях не каждый пиксель изображения несет полезную информацию. В некоторых системах нужно извлекать только соответствующие функции для целевого использования, что приводит ксжатию данных. Сжатие изображения выполняется в тот момент, когда датчик его захватывает [8]. Конструкции с использованием обычных многопиксельных массивов были реализованы 
с помощью камер с кодированной апертурой, интеграторов с коммутаторными конденсаторами [9] и сигма-дельта АЦП в каждом столбце. Такие датчики были опробованы для широкого спектра применений, но наиболее продуктивны, когда известно, что изображения являются разреженными, как в медицинских приложениях, военных (радарах) и астрономии.

\section{D-изображения}

Наряду с получением двумерного изображения в ряде приложений требуется информация о третьем пространственном измерении - глубине, а иногда нужно определить относительное расстояние до объектов, представленных в сцене. Эти приложения включают в себя виртуальную реальность, навигационную поддержку, топографию, биосенсоры и машинное зрение. Расстояние можно измерить по методу интерферометрии, триангуляции или времени пролета (Time-of-Flight, ToF) [10]. Meтод триангуляции использует геометрические измерения и может быть активным или пассивным (стереовидение). В пассивной форме система требует точной синхронизации двух или более сигналов фотоприемников, сложной обработки сигнала после захвата. LiDAR- (Light «radar») и TоF-изображения используют активный источник света для измерения расстояния до объекта. Этот источник света необходимо синхронизировать с датчиком. Чтобы не мешать двумерному изображению (при необходимости), источник света и детекторы работают в другом спектральном диапазоне, обычно ближнем инфракрасном.

\section{ОТЕЧЕСТВЕННЫЕ РАЗРАБОТКИ}

В России выполняются заказные разработки матричных КМОП-фотоприемников для различных потребителей в режиме fabless. В настоящее время одним из центров компетенций в данной области является ООО «Юник Ай Сиз» [11].

В активе компании - разработка и производство по КМОП-технологии с топологическими нормами 180 нм следующих типов фотоприемников с построчным считыванием (Rolling Shutter):

- матричные фотоприемники с разрешением $128 \times 128$ пикселей, которые отличаются высокой чувствительностью и кадровой частотой (до 2,5K fps), с обработкой видеоинформации внутри кристалла - для задач обнаружения и обработки динамических целей;

- матричные фотоприемники с разрешением $128 \times 128$ пикселей для систем дистанционного зондирования Земли;

- матричные фотоприемники с разрешением 1024 ×1024 для систем наблюдения и астронавигации;

- линейные фотоприемники считывания штриховых кодов.
Среди перспективных направлений - создание элементной базы однофотонных (лавинных) фотоприемников для систем интроскопии и дальнометрии (лидары); разработка матричных фотоприемников управляемого спектрального диапазона для медицинских систем (онкомаркеров, секвенаторов ДНК); матричные фотоприемники с разрешением 2048 × 2048 для систем наблюдения и астронавигации; разработка матричных фотоприемников с кадровым считыванием (Global Shutter) и с высокой кадровой частотой (fast CAM).

$$
\because *
$$

Впечатляющие достижения в конструкции и технологии изготовления КМОП-фотоприемников продолжаются. Наряду с улучшением их параметров и снижением стоимости уже ожидается выход на рынок однофотонных фотоприемников, многоэлементных органических фотоприемников на гибких подложках.

\section{ЛИТЕРАТУРА}

1. Carlos L., Gouveia P. Advances on CMOS image sensors // Sensor Review. V. 36. Issue: 3. P. 231-239.

2. Stefanov K.D., Clarke A.S., Ivory J., Holland A.D. Design and Performance of a Pinned Photodiode CMOS Image Sensor Using Reverse Substrate Bias // Sensors. 2018. V. 18. P. 118. doi:10.3390/s18010118.

3. Watanabe H. et al. A $1.4 \mu \mathrm{m}$ front-side illuminated image sensor with novel light guiding structure consisting of stacked lightpipes // IEDM. 2011. doi: 10.1109/IEDM.2011.6131513.

4. Krishnamurthy R. et al. Samsung S5K2P2XX 16 MP, $1.12 \mu \mathrm{m}$ Pixel Size Back-Illuminated ISOCELL CIS from the Samsung Galaxy S5 Smartphone Primary Camera Imager Process // Review. Chipworks. May 2014

5. Ahn J. et al. A 1/4-inch 8Mpixel CMOS Image Sensor with 3D BacksideIlluminated $1.12 \mu \mathrm{m}$ Pixel with Front-side Deep-Trench Isolation and Vertical Transfer Gate // ISSCC. 2014.

6. Nose A. et al. Design and Performance of a 1 ms High-Speed Vision Chip with 3D-Stacked 140 GOPS Column-Parallel PEs // Sensors. 2018. V. 18. P. 1313. doi:10.3390/s18051313.

7. Machado W. et al. Canon LCl290A (Die Markings) 20.2 Mp, $4.1 \mu \mathrm{m}$ Pixel Size Dual Pixel CMOS AF APS-C CMOS Image Sensor from the Canon EOS-70D (W) DSLR Camera Imager Process // Review. Chipworks. 2013. October.

8. Donoho D.L. Compressed sensing // IEEE Transactions on Information Theory. 2006. V. 52. No. 4. P. 1289-1306.

9. Dadkhah M., Deen M.J., Shirani S. Compressive sensing image sensors-hardware implementation // Sensors (Basel, Switzerland). 2013. V. 13. No. 4. P. 4961-4978.

10. Beer S., Seitz P. Real-time tomographic imaging without x-rays: a smart pixel array with massively parallel signal processing for real-time optical coherence tomography performing close to the physical limits // Research in Microelectronics and Electronics. 2005. Ph D.V. 2, IEEE. P. 135-138.

11. http://www.uniqueics.ru/ 